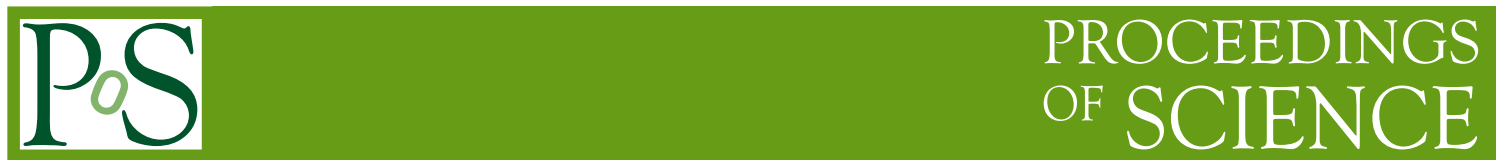

\title{
New photon results from CDF
}

\author{
Costas VELLIDIS*广 \\ FNAL \\ E-mail: vellidis@fnal.gov
}

\section{Ray Culbertson}

FNAL

E-mail: rlcefnal.gov

\section{Ting-Jun Yang}

FNAL

E-mail: tjyang@fnal.gov

We present results of the cross section measurement for photon production associated with bottom- and charm-quark production and of the cross section measurement for diphoton production. The measurements use the full CDF data sample and they are compared with state-of-the-art calculations. The comparisons show that subprocesses of order higher than next-to-leading in the coupling parameter of the strong interaction are important to describe the data accurately.

XXI International Workshop on Deep-Inelastic Scattering and Related Subjects 22-26 April, 2013

Marseilles, France

\footnotetext{
${ }^{*}$ Speaker.

$\dagger$ On behalf of the CDF Collaboration.
} 


\section{Introduction}

CDF has an extensive active program of prompt photon measurements using the full data set. The program benefits from the clean identification and precise measurement of the energy and direction of photons with the $\mathrm{CDF}$ detector. For the reported measurements, the candidate prompt photons are selected offline from tower clusters of the electromagnetic calorimeter and reconstructed in a cone of radius $\mathrm{R}=0.4$ in the $\eta-\phi$ plane. The photons are required to be central, $|y|<1$, and to have a transverse energy $E_{T}>30 \mathrm{GeV}$ in the photon+heavy flavor measurements and $E_{T}>17,15 \mathrm{GeV}$ (for the $1^{\text {st }}$ and $2^{\text {nd }}$ photon in the event, respectively) in the diphoton measurements. They are also required to be isolated in a cone of radius $\mathrm{R}=0.4$, with an isolation energy $<2$ $\mathrm{GeV}$. For the photon+heavy flavor measurements, a secondary vertex algorithm is used to select heavy flavor jets, which are required to have $|y|<1.5$ and $p_{T}>20 \mathrm{GeV} / c$. The charm and bottom fractions are derived by fitting simulated templates to the invariant mass distributions of the jets selected from the data. All measurements are corrected to parton level.

\section{Photon + heavy flavor production}

The measurements [1] are compared with parton-shower calculations from the PYTHIA [2] and SHERPA [3] Monte Carlo programs, with a next-to-leading order (NLO) calculation in the strong coupling parameter from [4], and with a $\mathrm{k}_{\mathrm{T}}$-factorization calculation from [5]. Predictions from PYTHIA are obtained for two cases: One with the default gluon splitting rates into heavy flavor quark pairs and one with these rates increased by a factor of 2 . The upper four windows of Figure 1 show the measured and predicted cross sections and the data/theory ratios differential in the photon transverse energy. A scale uncertainty is estimated for the NLO and $\mathrm{k}_{\mathrm{T}}$-factorization calculations. The ratios of the two PYTHIA calculations, default and $\times 2 \mathrm{~g} \rightarrow \mathrm{QQ}$, to the SHERPA calculation are shown together with the ratio of the data to the SHERPA calculation.

The default PYTHIA calculation describes the shapes rather well but underestimates the scale of the data by about a factor of 2 . The PYTHIA $\times 2 \mathrm{~g} \rightarrow \mathrm{QQ}$ calculation is significantly improved with respect to the default. However, neither this nor the SHERPA nor the NLO calculation reproduce all aspects of the data. The $\mathrm{k}_{\mathrm{T}}$-factorization calculation provides the best description of the data. This calculation accounts for off-mass shell subprocesses and uses unintegrated parton distributions to resum gluon radiation with $\mathrm{k}_{\mathrm{T}}^{2}$ above the matrix element scale $\mu^{2}$, which effectively incorporates contributions of order higher than NLO.

\section{Diphoton production}

The measurements [6] are compared with six calculations: predictions from the parton shower programs (i) PYTHIA [2] and (ii) SHERPA [3]; (iii) NLO predictions including non-perturbative fragmentation at leading order (LO) from the program MCFM [7]; (iv) NLO predictions including non-perturbative fragmentation at NLO from the program DIPHOX [8]; (v) predictions from the program RESBOS [9] that performs a low- $p_{T}$ analytically resummed calculation, which is then matched to the high- $p_{T}$ NLO matrix element calculation; and (vi) next-to-next-to-leading order (NNLO) predictions from [10]. PYTHIA is run both in the default mode and in a mode that com-

bines $\gamma \gamma$ and $\gamma+$ jet production $(\gamma \gamma+\gamma \mathrm{j})$ from which events with at least two prompt photons are 
selected during the simulation, thus including real NLO contributions from initial- and final-state radiation.

Figures 1 (the bottom two windows) and 2 show the measured and predicted cross sections and their relative deviations, in the form of (data-theory)/theory, differential in the diphoton transverse momentum $P_{T}$ and the azimuthal difference $\Delta \phi$ between the two photons in the event. Many more plots for other kinematic variables and different kinematic conditions can be found in the online edition of [6] as supplemental material. The PYTHIA $\gamma \gamma$ calculation fails entirely, but the $\gamma \gamma+\gamma$ j calculation is substantially improved. The NLO and NNLO calculations fail in the limit $P_{T} \rightarrow 0$. The RESBOS calculation provides the best description of the data in the limit of low diphoton $P_{T}$, where resummation is most important. The SHERPA calculation provides a reasonably good description of the data. The best overall agreement with the data is achieved by the NNLO prediction, thus showing the importance of higher-order subprocesses, although it tends to overestimate the high- $P_{T}$ part of the spectrum.

\section{Summary}

We report high precision measurements of the cross sections for prompt photon production associated with heavy quark flavor production and for prompt diphoton production, both using the full CDF data sample. The measurements are compared with state-of-the-art calculations. The comparisons show that subprocesses of order higher than next-to-leading in the coupling parameter of the strong interaction are important for the accurate description of the data.

\section{References}

[1] T. Aaltonen et al. (CDF Collaboration), arXiv:1303.6136, submitted to Phys. Rev. Lett.

[2] T. Sjöstrand, P. Eden, C. Friberg, L. Lombard, G. Miu, S. Mrenna, and E. Norrbin, Comp. Phys. Comm. 135, 238 (2001).

[3] T. Gleisberg, S. Höche, F. Kraus, M. Schönherr, S. Schumann, F. Siegert, and J. Winter, J. High Energy Phys. 02, 007 (2009).

[4] J. F. Owens and T. P. Stavreva, Phys. Rev. D 79, 054017 (2009).

[5] A. V. Lipatov, M. A. Malyshev, and N. P. Zotov, J. High Energy Phys. 1205, 104 (2012).

[6] T. Aaltonen et al. (CDF Collaboration), Phys. Rev. Lett. 110, 101801 (2013).

[7] J. M. Campbell and R. K. Ellis, Phys. Rev. D 60, 113006 (1999).

[8] T. Binoth, J. P. Guillet, E. Pilon,, and M. Werlen, Phys. Rev. D 63, 114016 (2001).

[9] C. Balazs, E. L.Berger, P. Nadolsky, and C.-P. Yuan, Phys. Rev. D 76, 013008 (2007).

[10] S. Catani, L. Cieri, D. de Florian, G. Ferra, and M. Grazzini, Phys. Rev. Lett. 108, 072001 (2012). 

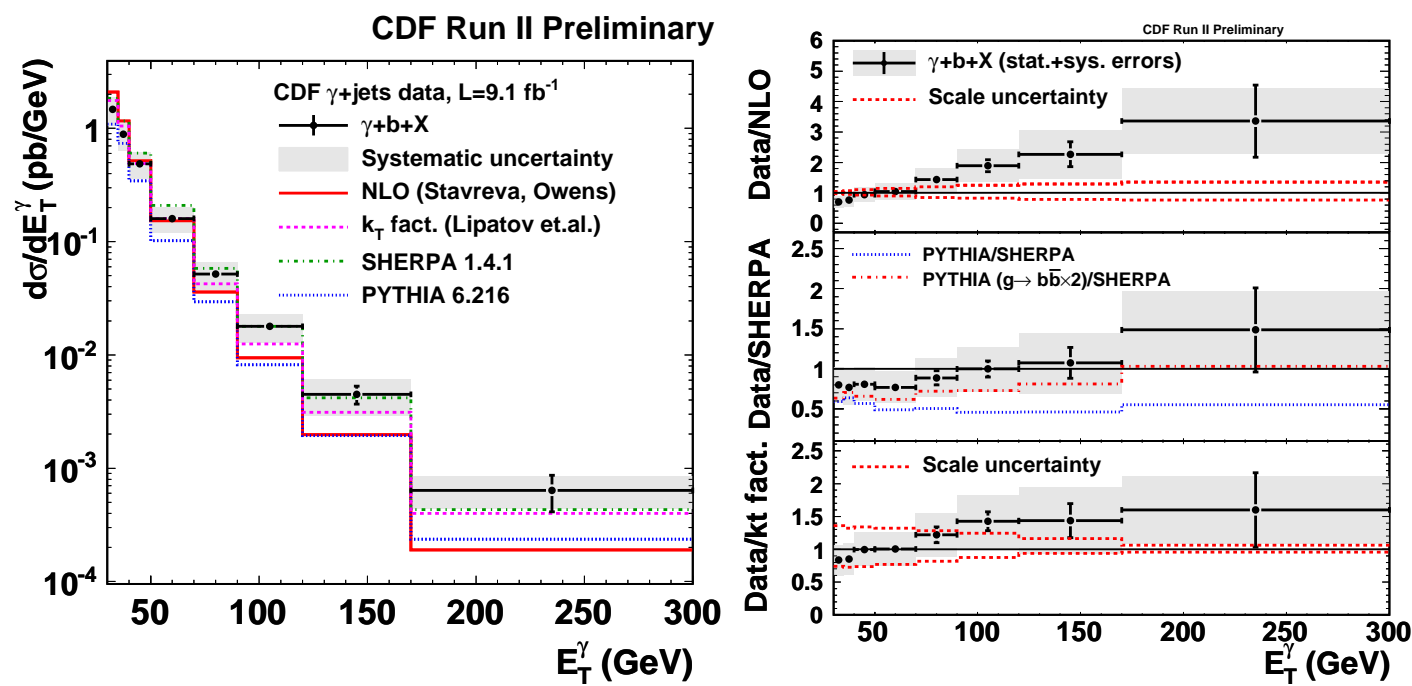

CDF Run II Preliminary
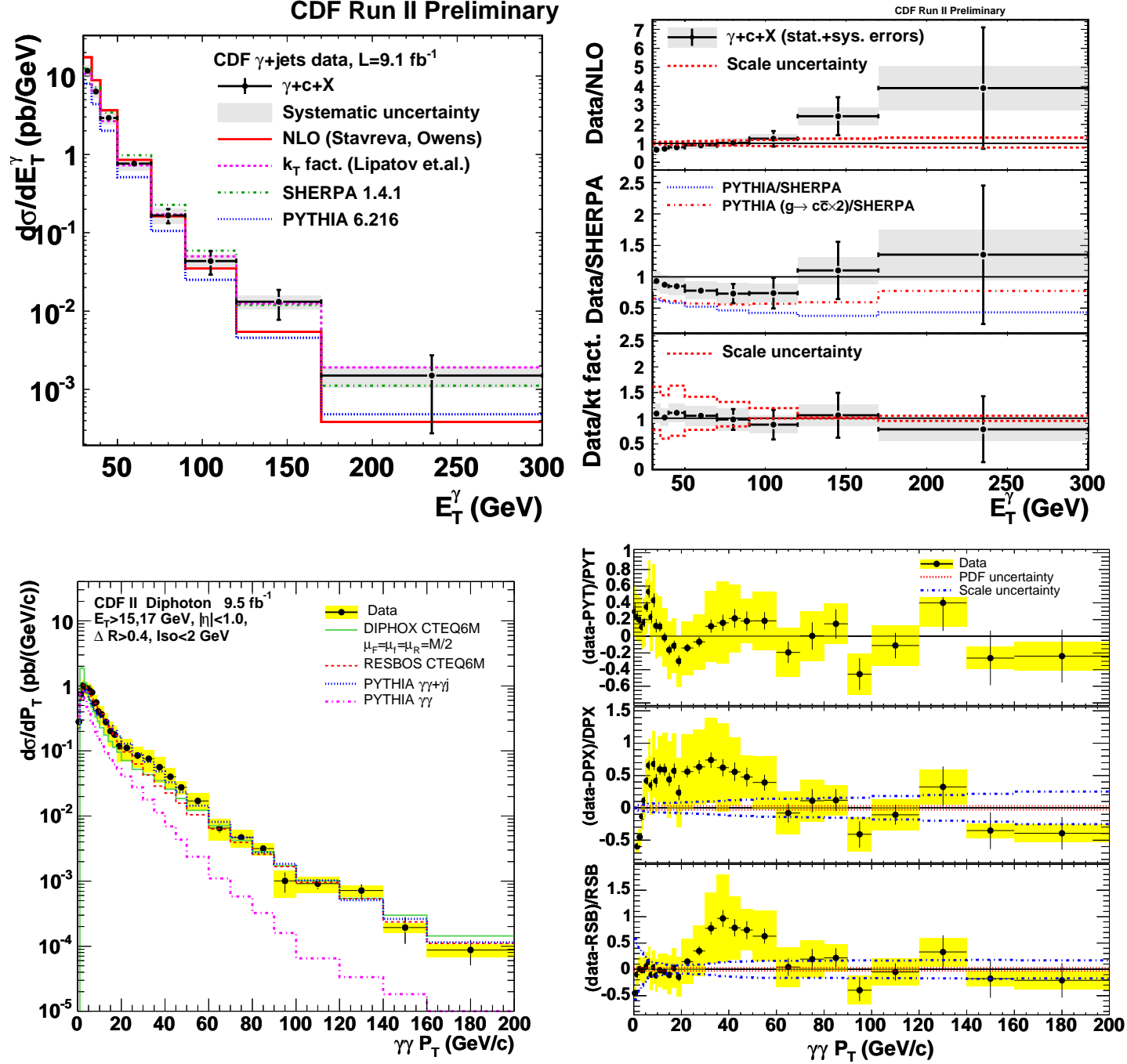

Figure 1: Differential cross sections and data-to-theory ratios for $\gamma+$ heavy flavor (upper four windows) and diphoton production. The shaded band shows the total systematic uncertainty of the measurement. 

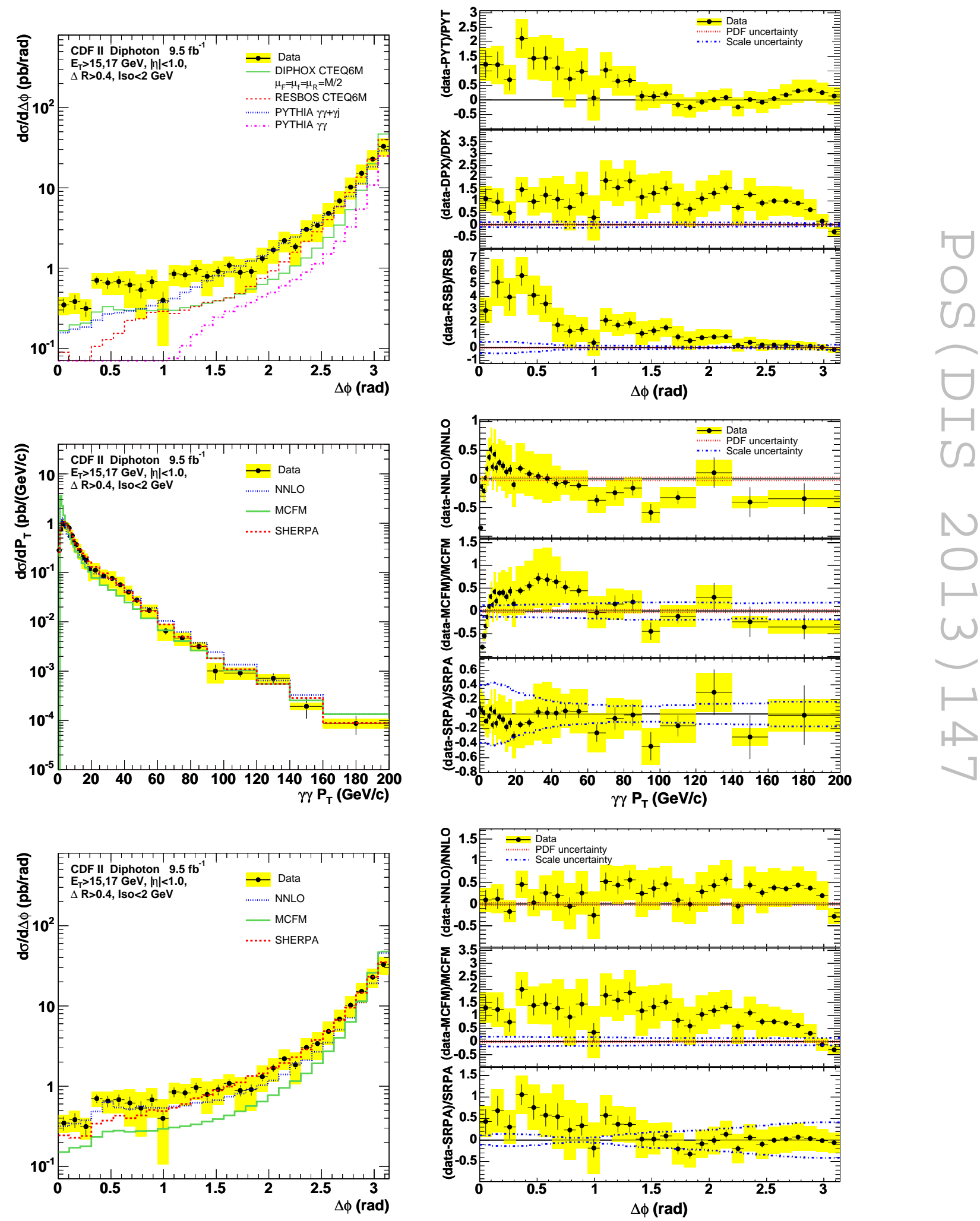

Figure 2: Differential cross sections and data-to-theory ratios for diphoton production. The shaded band shows the total systematic uncertainty of the measurement. 